\title{
Modeling of orientational polarization within the framework of extended micropolar theory
}

Received: 2 October 2020 / Accepted: 7 January 2021 / Published online: 28 January 2021

(C) The Author(s) 2021

\begin{abstract}
In this paper the process of polarization of transversally polarizable matter is investigated based on concepts from micropolar theory. The process is modeled as a structural change of a dielectric material. On the microscale it is assumed that it consists of rigid dipoles subjected to an external electric field, which leads to a certain degree of ordering. The ordering is limited, because it is counteracted by thermal motion, which favors stochastic orientation of the dipoles. An extended balance equation for the microinertia tensor is used to model these effects. This balance contains a production term. The constitutive equations for this term are split into two parts, one, which accounts for the orienting effect of the applied external electric field, and another one, which is used to represent chaotic thermal motion. Two relaxation times are used to characterize the impact of each term on the temporal development. In addition homogenization techniques are applied in order to determine the final state of polarization. The traditional homogenization is based on calculating the average effective length of polarized dipoles. In a non-traditional approach the inertia tensor of the rigid rods is homogenized. Both methods lead to similar results. The final states of polarization are then compared with the transient simulation. By doing so it becomes possible to link the relaxation times to the finally observed state of order, which in terms of the finally obtained polarization is a measurable quantity.
\end{abstract}

\section{Polarization in dielectrics modeled by extended micropolar theory}

Extended micropolar theory is capable of modeling structural changes in materials. The orientational polarization of a dielectric can be considered as a structural change and is, therefore, a suitable candidate for this kind of modeling. The paper is organized as follows. In this section we will present the basic relations of micropolar theory, first, in general terms, and then, in particular, focus on a discussion of its extension. The next section will, first, mostly for the benefit of the mechanics community, provide a short recapitulation of the phenomenon of electric polarization, in particular orientational polarization. Second the equations of extended micropolar theory will be specialized to the requirement of its modeling. In particular we shall specify the required production term based on physical grounds. Moreover, Sect. 2 presents homogenization procedures that allow to capture the final state of polarization. These results can then be linked to the modeling of the

Communicated by Marcus Aßmus, Victor A. Eremeyev and Andreas Öchsner.

E. N. Vilchevskaya

Institute for Problems in Mechanical Engineering of the Russian Academy of Sciences, Bol'shoy pr. 61, V.O., 199178 St. Petersburg, Russia

W. H. Müller ( $\varangle)$

Institute of Mechanics, Chair of Continuum Mechanics and Constitutive Theory, Technische Universität Berlin, Sekr. MS. 2, Einsteinufer 5, 10587 Berlin, Germany

E-mail: wolfgang.h.mueller@tu-berlin.de 
temporal evolution of polarization obtainable within the framework of extended micropolar theory. The paper will end with a summary and an outlook into further applications based on the presented results.

\subsection{Introductory remarks: Benefits of the concept of the microinertia tensor}

Traditionally the microinertia tensor of a continuum particle, $\boldsymbol{J}$, plays an important role only in context with its rotational degrees of freedom. In combination with the angular velocity vector, $\omega$, it characterizes the spin of the continuum element. The details are outlined in Eringen's theory of micropolar media, see for example [11]. There it is shown that the microinertia tensor obeys a kinematic constraint in form of a rate equation, which expresses the possibility of material continuum particles to undergo rigid body rotations. This feature is captured by means of three rotating rigid directors. Within the framework of this theory the shape of the microinertia tensor does not change; rather, it can only rotate rigidly. Eringen calls such materials micropolar media. However, as we shall demonstrate in this paper, describing the particle spin is not the only use of the microinertia tensor. If generalized, the concept of a changing microinertia can be beneficial for describing processes in certain materials, for example, electromagnetic ones, such as the development of electric polarization, which may already occur under the absence of an angular velocity. Some other applications of micropolar theory can be found, for example, in $[3,4]$.

In fact a radical change of the concept of the microinertia tensor has been presented recently in [14]. There the microinertia tensor is treated as a completely independent field variable for solid and fluid matter alike. In this formulation closed as well as open systems are allowed. This means that in- and outflux of matter in a Representative Volume Element (RVE) can be taken into account and the concept of a material particle is not imperative. Moreover, a structural change due to external forces becomes possible. The microinertia tensor becomes a fully independent field variable with its own balance requiring additional constitutive quantities. More specifically, in contrast with the balance of mass, the balance for the micro-inertia tensor is not conserved. It contains a production term, $\chi$, which could be specified by following the rules of constitutive theory or be motivated by physics and intuition, such that fundamental principles are not violated.

In the following subsections it will be demonstrated that this extended theory allows for the modeling of processes accompanied by a considerable structural change characterized by a changing microinertia within a representative volume element, such as the development of orientational polarization in matter under the action of an external electric field, $\boldsymbol{E}$. In this context the multi-disciplinary aspect of the present formulation should be stressed. Potentially it can be used fruitfully to synthesize new innovative materials [34], which combine mechanical and electrical behavior. For example, the use of piezoelectric patches can provide reduction in vibrations or energy harvesting (see, e.g., $[2,12]$ ). Moreover, for a recent thermodynamically consistent treatment of electro-mechanical problems see [1].

\subsection{The balances of micropolar media}

The motion and state of micropolar media in spatial description are described by the following coupled system of differential equations:

- balance of mass,

$$
\frac{\delta \rho}{\delta t}=-\rho \nabla \cdot \boldsymbol{v},
$$

- balance of momentum,

$$
\rho \frac{\delta \boldsymbol{v}}{\delta t}=\nabla \cdot \boldsymbol{\sigma}+\rho \boldsymbol{f}
$$

- balance of spin,

$$
\rho \boldsymbol{J} \cdot \frac{\delta \boldsymbol{\omega}}{\delta t}=-\rho \boldsymbol{\omega} \times \boldsymbol{J} \cdot \boldsymbol{\omega}+\nabla \cdot \boldsymbol{\mu}+\boldsymbol{\sigma}_{\times}+\rho \boldsymbol{m},
$$

- balance of internal energy,

$$
\rho \frac{\delta u}{\delta t}=\boldsymbol{\sigma}:(\nabla \otimes \boldsymbol{v}+\mathbf{I} \times \boldsymbol{\omega})+\boldsymbol{\mu}: \nabla \otimes \boldsymbol{\omega}-\nabla \cdot \boldsymbol{q}+\rho r,
$$


where $\rho$ is the field of mass density, $\boldsymbol{v}$ and $\boldsymbol{\omega}$ are the linear and angular velocity fields, $\boldsymbol{\sigma}$ is the non-symmetric Cauchy stress tensor, $\boldsymbol{f}$ is the specific body force, $\boldsymbol{J}$ is the specific micro-inertia tensor, $\boldsymbol{\mu}$ is the non-symmetric couple stress tensor, $(\boldsymbol{a} \otimes \boldsymbol{b})_{\times}=\boldsymbol{a} \times \boldsymbol{b}$ is the Gibbsian cross, $\boldsymbol{m}$ are specific body couples, $u$ is the specific internal energy, $\boldsymbol{q}$ is the heat flux, and $r$ is the specific heat supply. By the colon we denote the outer double scalar product between tensors of second rank, $\boldsymbol{A}: \boldsymbol{B}=A_{i j} B_{i j}$. Moreover,

$$
\frac{\delta(\cdot)}{\delta t}=\frac{\mathrm{d}(\cdot)}{\mathrm{d} t}+(\boldsymbol{v}-\boldsymbol{w}) \cdot \nabla(\cdot)
$$

is the substantial derivative of a field quantity, $\mathrm{d}(\cdot) / \mathrm{d} t$ the total derivative, and $\boldsymbol{w}$ the mapping velocity of the observational point (see [13]).

It was already indicated that traditional micropolar theory assumes that each material point or "particle" of a micropolar continuum is phenomenologically equivalent to a rigid body. It can rotate but the state of the rotational inertia in the principal axes system does not change. In other words the micro-inertia tensor will not change its form nor shape, see, for example, [9], [32], [19], [11]. Even if a so-called micromorphic medium is considered, which in principle allows an intrinsic change of micro-inertia (following [8], [11], [10]), many publications use only the following additional equation for the conservation of inertia (e.g., see [23], [5]), which is an identity of rigid body kinematics:

$$
\frac{\delta \boldsymbol{J}}{\delta t}=\omega \times \boldsymbol{J}-\boldsymbol{J} \times \boldsymbol{\omega} .
$$

Note again that the terms on the right-hand side characterize the change of the inertia tensor, which is exclusively due to rigid body rotation.

An extension to this approach was suggested in [7], where it was proposed that the microinertia of polar particles may change as the continuum deforms. This idea was then further elaborated in [14], where it was clearly stated that the tensor of microinertia should be treated as an independent field. Within that approach a fixed and open elementary volume $V$ was treated as a micropolar continuum (macro-) region, as it is customarily done in spatial description. Then its microinertia tensor $\boldsymbol{J}$ (in units $\mathrm{of}^{2}$ ) as a property on the continuum scale is obtained by homogenization as follows. Within the elementary volume $V$ there are $i=1, \ldots, N$ microparticles of mass $m_{i}$ and inertia tensor $\hat{\boldsymbol{J}}_{i}$ (in units of $\mathrm{kgm}^{2}$ ) such that:

$$
m=\frac{1}{N} \sum_{i=1}^{N} m_{i}, \quad \boldsymbol{J}=\frac{1}{N m} \sum_{i=1}^{N} \hat{\boldsymbol{J}}_{i}
$$

where $m$ is the average mass within $V$. If the linear and angular velocities of the particles are denoted by $\boldsymbol{v}_{i}$ and $\omega_{i}$, then the specific linear and angular momenta are given by:

$$
\boldsymbol{v}=\frac{1}{N m} \sum_{i=1}^{N} m_{i} \boldsymbol{v}_{i}, \quad \boldsymbol{J} \cdot \boldsymbol{\omega}=\frac{1}{N m} \sum_{i=1}^{N} \hat{\boldsymbol{J}}_{i} \cdot \boldsymbol{\omega}_{i} \Rightarrow \boldsymbol{\omega}=\left(\sum_{i=1}^{N} \hat{\boldsymbol{J}}_{i}\right)^{-1} \cdot \sum_{i=1}^{N} \hat{\boldsymbol{J}}_{i} \cdot \boldsymbol{\omega}_{i} .
$$

The specific linear momentum is nothing else but the translational velocity on the continuum scale. Equation $(1.8)_{1}$ simplifies considerably if all the microparticles have the same mass:

$$
\boldsymbol{v}=\frac{1}{N} \sum_{i=1}^{N} \boldsymbol{v}_{i}
$$

This will be the case for the dielectric medium considered in this paper. Moreover, we will also assume that the inertia tensors of the microparticles are the "same." This means that the three principal values of the inertia tensor are the same for each particle, $\hat{J}_{i}^{j}=\hat{J}^{j}$, but its eigensystem vectors $\boldsymbol{e}_{i, j}^{*}, j=1,2,3$ are not, because the microparticles are randomly oriented. Thus, in such a case we can only say that

$$
\hat{\boldsymbol{J}}_{i}=\hat{J}^{1} \boldsymbol{e}_{i, 1}^{*} \otimes \boldsymbol{e}_{i, 1}^{*}+\hat{J}^{2} \boldsymbol{e}_{i, 2}^{*} \otimes \boldsymbol{e}_{i, 2}^{*}+\hat{J}^{3} \boldsymbol{e}_{i, 3}^{*} \otimes \boldsymbol{e}_{i, 3}^{*} \Rightarrow \boldsymbol{J} \neq \frac{\hat{\boldsymbol{J}}_{i}}{m}
$$


Only for a spherical inertia tensor, or if all microparticles are aligned in the same manner, the last relation would turn into an equality. This also means that in all other cases of "equal" inertia tensors we must conclude from the last relation in (1.8) that

$$
\boldsymbol{\omega} \neq \frac{1}{N} \sum_{i=1}^{N} \boldsymbol{\omega}_{i} .
$$

Because of the movement of the medium, the elementary volume contains different microparticles as time passes, and the microinertia tensor assigned to the volume will change due to the incoming or outgoing flux of inertia. However, internal structural transformations are also possible. These can be due to (a) the combination or fragmentation of the particles during mechanical crushing, to (b) chemical reactions, or to (c) changes of the anisotropy of the material, for example by applying external electromagnetic fields. Such effects are explained in greater detail in [22], [21], [20], or [33]. In a nutshell, on the continuum scale all of this can be taken into account by adding a source or production term, $\chi$, to the right-hand side of Eq. (1.6), which now reads:

$$
\frac{\delta \boldsymbol{J}}{\delta t}=\omega \times \boldsymbol{J}-\boldsymbol{J} \times \omega+\chi .
$$

On the continuum level this source term must be considered as a new constitutive quantity for which an additional constitutive equation has to be formulated. The form of the constitutive equation depends on the problem under consideration and can be a function of many physical quantities. A suitable form for the modeling of orientational polarization in polarizable matter under the influence of an external electric field will be discussed in the next section.

Finally it should be emphasized once more that the field of microinertia, i.e., the rotational inertia of the continuum influences the development of the angular velocity $\omega$. The temporal development is dictated by the spin balance (1.3), and this is usually the only purpose of $\boldsymbol{J}$. In this paper it is different: Because of the extended balance (1.12) $\boldsymbol{J}$ can also be used to characterize structural changes of the micropolar medium, without the presence of an angular velocity. We proceed to explain this in more detail in the next section.

\section{Polarization modeling}

\subsection{Introductory remarks on polarization}

For didactical reasons we recapitulate a few facts from electrical engineering in this section. In this field one distinguishes between electrically conducting and non-conducting or insulating materials. On a microscopic scale the former possess freely movable electric charges, for example the electron gas in metals. In case of the latter charges cannot move around freely. Positive and negative charges must stay together. They are bound within a molecule or other basic atomic units, for example within a crystal lattice. Materials in which an electric current cannot flow are also known as dielectrics. In the absence of external electric fields they are electrically neutral. However, one of their basic properties is the ability to polarize if an external electric field $\boldsymbol{E}$ is applied. This leads to the creation of surface charges, which on the continuum scale are described by the polarization vector $\boldsymbol{P}$. Indeed, $\oint_{\partial V} \boldsymbol{P} \cdot \boldsymbol{n} \mathrm{d} A$ allows to compute that charge, provided the field $\boldsymbol{P}$ is known.

The question arises how the polarization can be measured, at least in principle, since a direct measurement of charges, in particular surface charges, is difficult. A simple school experiment can be used. Consider a plate capacitor, which is first charged by a battery, so that the plates of surface $A$ at a distance $d$ are loaded with the electric charges $\pm Q$. The battery is then detached and replaced by a voltmeter. If there is vacuum in between the plates, the voltmeter will show a voltage $U_{\text {vac }}$, say. If we now place a dielectric body in between this voltage decreases down to $U_{\mathrm{pol}}<U_{\mathrm{vac}}$. The stronger the decrease, the more surface charges are created, and the stronger the polarization $\boldsymbol{P}$ will be. In order to quantify the effect in terms of a material constant, let us assume for simplicity that the dielectric material between the plates is isotropic and the polarization vector can be described by a linear constitutive equation of the type:

$$
\boldsymbol{P}=\varepsilon_{0} \chi \boldsymbol{E},
$$

where $\varepsilon_{0}=8.854 \times 10^{-12} \frac{\mathrm{As}}{\mathrm{Vm}}$ is the electric field constant and $\chi>0$ is the dielectric susceptibility, which in this simple case is a material-dependent, dimensionless constant. In fact, application of the static Maxwell 
(a)

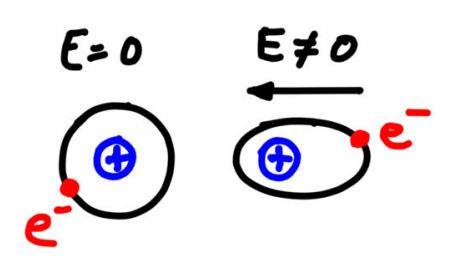

(b)

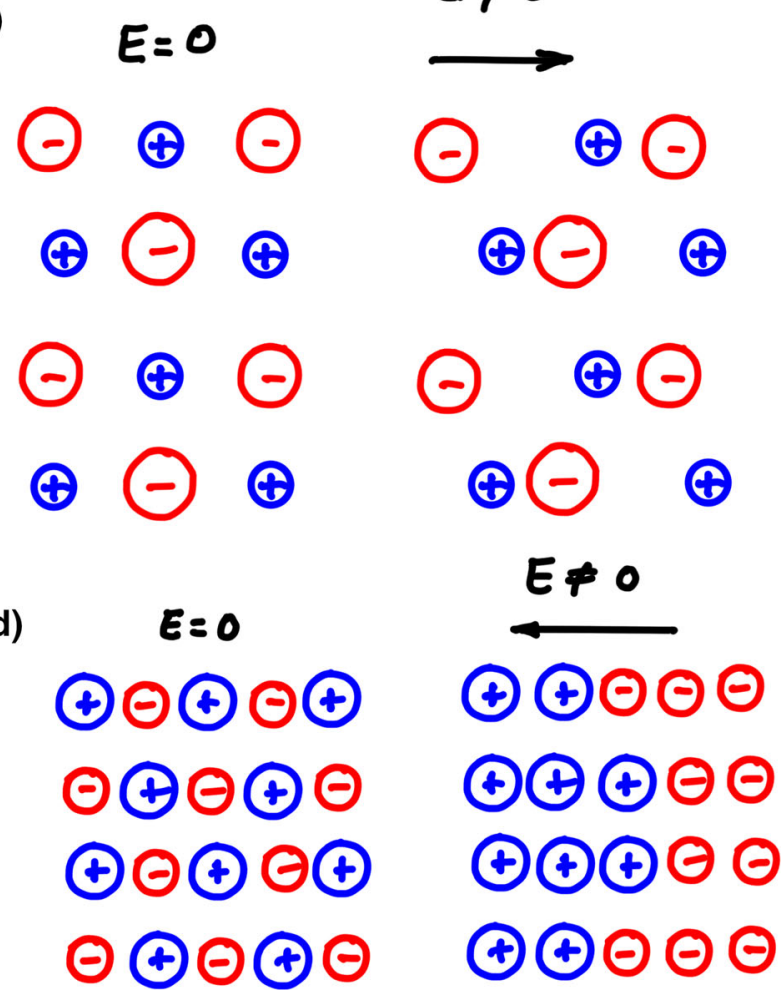

Fig. 1 Polarization types adapted after [16]

equations to the case of the plate capacitor described above yields:

$$
U_{\mathrm{pol}}=\frac{Q d}{\varepsilon_{0}(1+\chi) A} .
$$

The combination $\varepsilon_{r}=1+\chi$ is also known as relative permittivity, which is also dimensionless. It is equal to one for the case of vacuum. Then Eq. (2.2) allows to determine $\varepsilon_{r}$ or $\chi$ if the the two voltages before and after filling the vacuum are measured:

$$
\chi=\frac{U_{\mathrm{vac}}}{U_{\mathrm{pol}}}-1 .
$$

Note that if the dielectric is anisotropic and if the electric field is not too high, the dielectric susceptibility constant can simply be replaced by a constant second rank dielectric susceptibility tensor, $\chi$. For large electric fields the dielectric susceptibility is a nonlinear function of the electric field, $\chi(\boldsymbol{E})$, which is sometimes expressed in a power series. Moreover, analogously to viscoelasticity, which makes use of a frequency-dependent complex shear modulus during harmonic loading, it becomes necessary to introduce a frequency-dependent and complex valued dielectric susceptibility when harmonically alternating electric fields are applied.

It should be emphasized that we made these remarks just to illustrate the measurement principle. In practice (frequency dependent) susceptibilities and relative permittivities are measured dynamically, for example, by using microwave waveguide systems, see [35].

Figure 1 presents cartoons of the various polarization mechanisms encountered in materials on the microscale.

Inset (a) refers to electronic polarization. Due to the electric field the positive charge of the atomic nucleus and the negative charge of the surrounding electron cloud are shifted with respect to each other so that an atomic dipole results. If the field is removed, the electron cloud and the nucleus move reversibly into their old position. The atom is electrically neutral and no longer a dipole. It is a very weak effect in (more or less 
isolated) atoms or ions with spherical symmetry. It can be a strong effect in, e.g., covalently bonded materials, such as Silicon, Germanium or diamond [17,24].

Inset (b) illustrates ionic polarization. It emerges due to a shift in the positive and negative ion centers of gravity. A typical example are sodium chloride crystals [31].

Inset (c) illustrates orientational polarization, which will be modeled in this paper. It results from field stimulated orientation of atomistic aggregates that already carry a dipole moment before the external electric field is switched on. It is pertinent to polar liquids, but it can also be observed in solid polar organic substances. In this case, the polarization is usually not caused by the rotation of the molecule itself, but of the polar radicals present within it in relation to the molecule. In this context hydrochloric acid or water should be mentioned, because the charge distributions in these molecules are skewed so that a net permanent dipole moment arises. Moreover, in cellulose, the polarity is explained by the presence of $\mathrm{OH}$ and oxygen hydroxyl groups. In addition, crystals with a molecular lattice and weak van der Waals bonds can also orientate larger particles. A typical engineering example of materials that show orientational polarization are electrets [16].

Finally inset (d) presents spatial (or space) charge polarization. It is observed in dielectrics with a heterogeneous interlayer structure. Hence, it occurs when there is an accumulation of charge at an interface between two materials or between two regions within a material because of the external field. This can occur in compound dielectrics, or when there are two electrodes connected to a dielectric material [30].

It should be noted that the measurable dielectric constant is usually the result of several microprocesses that differ in the way and time at which a stationary state is reached. In particular, a distinction must be made between deformation (elastic) and thermal (relaxation) polarization. Elastic polarization relates to the rotation of molecules with constant dipoles relative to the equilibrium position under the influence of an external electric field. Dipole-elastic polarization is characteristic of those types of polar dielectrics in which the dipole moments of molecules cannot change their orientation significantly, but only oscillate with a small amplitude relative to the equilibrium position. In this case, the dipoles must be sufficiently rigidly coupled so that an elastic restoring force arises when the direction of orientation changes. This type of polarization is characteristic of liquid crystals and pyroelectrics. We shall not take elastic polarization into account in the model we are about to present and leave this to future work.

Thermal polarization is observed in dielectrics that contain weakly bound polar molecules that can move randomly during thermal motion. An external electric field leads to some order in the particle orientation, but, in general, thermal motion prevents the creation of a totally ordered orientation of all dipoles. Only at extremely low temperatures all the dipoles may be be aligned along the lines of force. Thus, only a partial orientation of the electric dipoles occurs under the influence of the field, i.e., depending on the strength of the electric field the dipoles tend to align toward it more or less but not completely (see Fig. 2, top right). We will now look into this in more detail.

\subsection{The microscopic and the continuum viewpoint}

The pictures in Fig. 2 relate to our model and show an RVE of matter capable of orientational polarization. The picture on the top left illustrates the situation before an external electric field was applied. We assume that on the microscopic or atomistic scale the material consists of rigid rods with positive and negative charges $q$ at their ends, such that the dipole moment of one rod is given by $\boldsymbol{p}=q \boldsymbol{l}$, where the length vector $\boldsymbol{l}$ points from the negative to the positive head. The dipoles are chaotically oriented. Therefore, the total dipole moment on a continuum scale is zero. We also refer to it as the averaged or homogenized polarization vector of the ensemble of rods within the RVE, $\langle\boldsymbol{p}\rangle$. In the literature this quantity is sometimes also referred to as micropolarization. When multiplied by the continuum field of particle density $n(\boldsymbol{x}, t)=N / V$ we obtain the aforementioned local polarization vector $\boldsymbol{P}=n\langle\boldsymbol{p}\rangle$.

Moreover, note that due to the requirement of isotropy before the electrical field acts, the microinertia tensor, $\boldsymbol{J}$, which is also a continuum quantity, must be a spherical tensor, see Fig. 2, bottom left, which unlike $\boldsymbol{P}$ is not zero.

Under the influence of an external electric field, $\boldsymbol{E}=E \boldsymbol{n}$, the polarized microparticles, i.e., the rigid rods, tend to align in the direction $\boldsymbol{n}$ of that field, in order to reduce the electrostatic energy of the material: Fig. 2, top right, $\boldsymbol{n}$ being the unit vector in electric field direction. Then the substance will carry a dipole moment on the continuum scale, which is no longer equal to zero, and which coincides with the direction of the electric field vector, $\langle\boldsymbol{p}\rangle=\langle p\rangle \boldsymbol{n} \neq \mathbf{0}$. Moreover, the microinertia tensor will change from spherical to transversal anisotropy in the direction of $\boldsymbol{n}$, as indicated in Fig. 2, bottom right. 


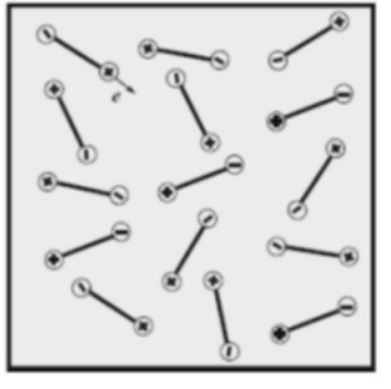

II

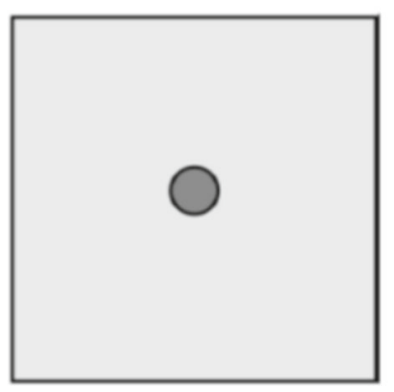

Fig. 2 Structural changes and their corresponding averaged properties

The idea is to model the time development of homogenized polarization, $\langle\boldsymbol{p}\rangle=q\langle\boldsymbol{l}\rangle(t)$, through the time development of the microinertia, $\langle\boldsymbol{J}\rangle=\langle\boldsymbol{J}\rangle(t)$, which will be used to compute a homogenized dipole length, $\langle\boldsymbol{l}\rangle=\langle\boldsymbol{l}\rangle(t)$. In order to capture this time development the kinetic equation (1.12) may serve. Of course, for its integration the production term needs to be specified, so that it mimics thermal polarization. These issues will be clarified in the next subsection by presenting a suitable mathematical framework.

\subsection{Compilation of relevant inertia tensor expressions and homogenization}

Recall from dynamic textbooks the inertia tensor with respect to the center of gravity per unit mass for a rigid rod of length $l$ oriented in $\boldsymbol{e}_{3}^{*}$-direction of its normalized eigenbase $\boldsymbol{e}_{i}^{*}, i=1,2,3$ :

$$
\boldsymbol{J}^{\mathrm{r}}=\frac{l^{2}}{12}\left(\boldsymbol{e}_{1}^{*} \otimes \boldsymbol{e}_{1}^{*}+\boldsymbol{e}_{2}^{*} \otimes \boldsymbol{e}_{2}^{*}\right) .
$$

For future calculations we decompose this expression into a spherical and into a deviatoric part:

$$
\boldsymbol{J}^{\mathrm{r}}=\boldsymbol{J}^{\mathrm{r}, \mathrm{sp}}+\boldsymbol{J}^{\mathrm{r}, \mathrm{dev}}, \quad \boldsymbol{J}^{\mathrm{r}, \mathrm{sp}}=\frac{l^{2}}{18} \mathbf{I}, \quad \boldsymbol{J}^{\mathrm{r}, \mathrm{dev}}=\frac{l^{2}}{36}\left(\mathbf{I}-3 \boldsymbol{e}_{3}^{*} \otimes \boldsymbol{e}_{3}^{*}\right)
$$

Clearly this is an expression related to the microscopic scenario. It holds for each microparticle shown in Fig. 2, top left. However, it can also be used to characterize the situations on the continuum level shown on the bottom right of that figure. The key to this is homogenization by averaging over the particle population. For this purpose we introduce the so-called probability density for transversal anisotropy, $P(\xi, \vartheta)$ as follows (see [15], Section 5.3.5 for variations in the equation in the case of a semi-sphere):

$$
P(\xi, \vartheta)=\frac{1}{2 \pi}\left(\left(1+\xi^{2}\right) \exp (-\xi \vartheta)-\frac{1}{2} \exp (-\xi \pi)\right), \quad 0 \leq \vartheta \leq \pi,
$$

$\xi$ is the so-called positive scatter parameter. Two limit cases are important: $\xi=0$, which characterizes fully random alignment of the rods, and $\xi=\infty$, where all rods are perfectly aligned in the direction $\boldsymbol{n}$ of the external 


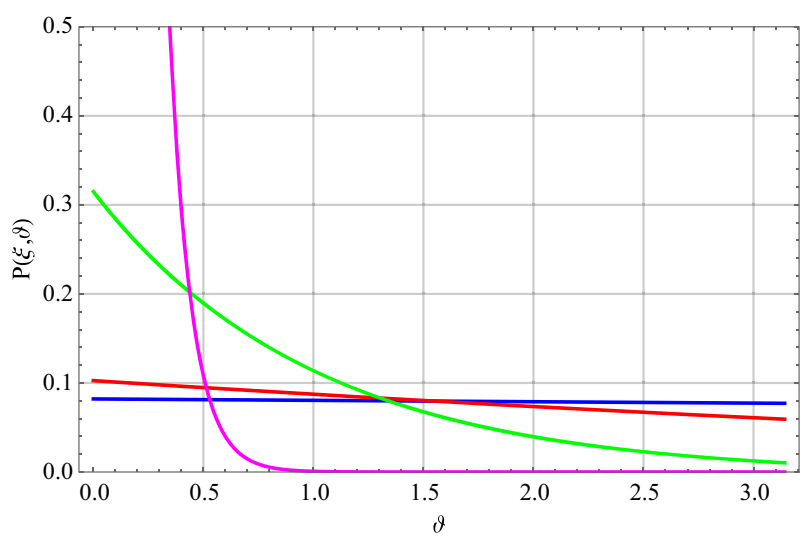

Fig. 3 Probability density function for $\xi=0.01$ (blue), $\xi=0.1$ (red), $\xi=1$ (green), $\xi=10$ (magenta)

electric field. $0 \leq \vartheta \leq \pi$ is the polar angle of the unit sphere $\Omega$. The probability density is normalized with respected to integration over $\Omega, 0 \leq \varphi \leq 2 \pi$ being the azimuthal angle:

$$
\int_{\Omega} P(\xi, \vartheta) \sin \vartheta \mathrm{d} \vartheta \mathrm{d} \varphi=1
$$

as can be demonstrated easily by using computer algebra programs. It is illustrated for different choices of $\xi$ in Fig. 3. The case $\xi=0$ corresponds to a constant value of $P$, such that all direction $\vartheta$ are equally possible. However, even for relatively small positive values of $\xi=0$ one departs from this case and runs into transversal anisotropy, which favors small values of $\vartheta$.

We will use it now for homogenization. In the first place we want to calculate the average length vector $\left\langle\boldsymbol{l}_{\infty}\right\rangle$ of the ensemble shown in Fig. 2, top right. The index $\infty$ is supposed to indicate that this is the effective, homogenized length after sufficient time has passed since the electric field was switched on. The directed length of an arbitrary rigid rod is given by $\boldsymbol{l}=\boldsymbol{l} \boldsymbol{e}_{3}^{*}$. However, the unit vector $\boldsymbol{e}_{3}^{*}$ is now arbitrarily oriented in space. In order to emphasize this point we assign $\boldsymbol{e}_{3}^{*} \rightarrow \boldsymbol{N}, \boldsymbol{N}$ being an arbitrarily oriented unit vector. To make this even more obvious we recall the representation of the unit vector $N$ in spherical coordinates,

$$
\boldsymbol{N}=\cos \varphi \sin \vartheta \boldsymbol{e}_{1}+\sin \varphi \sin \vartheta \boldsymbol{e}_{2}+\cos \vartheta \boldsymbol{e}_{3},
$$

where $\varphi$ and $\vartheta$ can vary as indicated before, so that all directions in space are addressed by points on the unit sphere. The vector $\boldsymbol{N}$ is spanned with respect to a special Cartesian base $\boldsymbol{e}_{i}, i=1,2,3$ located in the center of the RVEs shown in Fig. 2, such that the vector $\boldsymbol{e}_{3}$ is oriented in the direction of the external electric field, $\boldsymbol{n}$. Hence we write $\boldsymbol{e}_{3}=\boldsymbol{n}$, and $\boldsymbol{e}_{1}$ and $\boldsymbol{e}_{2}$ are arbitrarily oriented perpendicularly to it within the corresponding plane of isotropy. Then $\left\langle\boldsymbol{l}_{\infty}\right\rangle$ can be calculated by averaging as follows:

$$
\left\langle\boldsymbol{l}_{\infty}\right\rangle=\int_{\Omega} P(\xi, \vartheta) \boldsymbol{l} \mathrm{d} \Omega=l \int_{\vartheta=0}^{\pi} \int_{\varphi=0}^{2 \pi} P(\xi, \vartheta) \boldsymbol{N}(\vartheta, \varphi) \sin \vartheta \mathrm{d} \vartheta \mathrm{d} \varphi=\frac{\left(1+\xi^{2}\right)(1-\exp (-\xi \pi))}{4+\xi^{2}} \ln ,
$$

$\mathrm{d} \Omega$ being the surface element of the unit sphere. Clearly within the plane of isotropy no average length contribution must arise. Everything points in the direction of transversal anisotropy, $\boldsymbol{n}$. Note that for the case of perfect disorder $\xi=0$ we obtain zero effective length, as expected. Moreover, for perfect alignment $\xi=\infty$ the homogenization yields $\left\langle\boldsymbol{l}_{\infty}\right\rangle=\boldsymbol{l} \boldsymbol{n}$ as could have also be expected. We can use (2.9) to compute the effective final dipole moment on the continuum scale from $\left\langle\boldsymbol{p}_{\infty}\right\rangle=q\left\langle\boldsymbol{l}_{\infty}\right\rangle$. However, note once more that all these homogenizations characterize only the final state. The temporal development of how to get from the initially isotropic to the non-isotropic state cannot be analyzed within this approach.

Next we homogenize the inertia tensor (2.5) of partially aligned rods within the RVE. To this end we assign $\boldsymbol{e}_{3}^{*} \rightarrow \boldsymbol{N}$ and carry out various integrations by using computer algebra programs. The final result reads:

$$
\left\langle\boldsymbol{J}_{\infty}\right\rangle=\int_{\Omega} P(\xi, \vartheta) \boldsymbol{J}^{\mathrm{r}} \mathrm{d} \Omega=\left\langle\boldsymbol{J}_{\infty}^{\mathrm{sp}}\right\rangle+\left\langle\boldsymbol{J}_{\infty}^{\mathrm{dev}}\right\rangle
$$




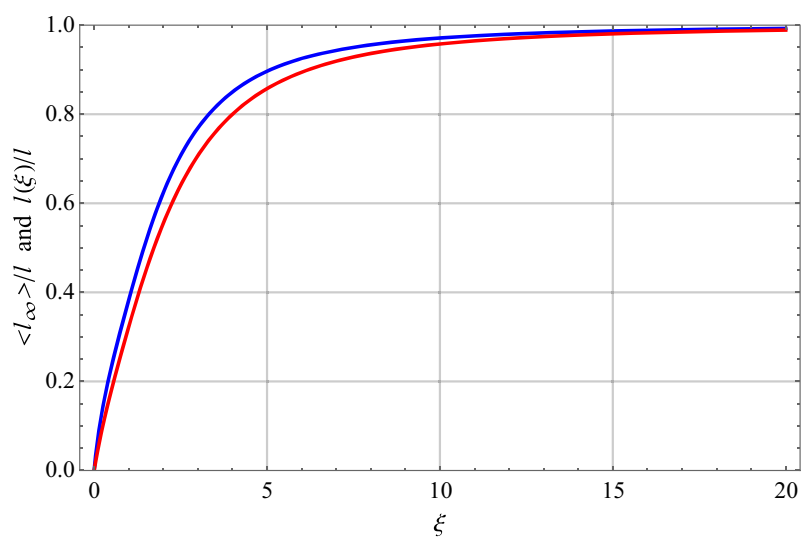

Fig. 4 Dependence of the normalized dipole moment from length homogenization (blue line) and from microinertia (red line) on the scattering parameter

with an average spherical and an average deviatoric part,

$$
\left\langle\boldsymbol{J}_{\infty}^{\mathrm{sp}}\right\rangle=\frac{l^{2}}{18} \mathbf{I}, \quad\left\langle\boldsymbol{J}_{\infty}^{\mathrm{dev}}\right\rangle=\frac{\bar{l}(\xi)^{2}}{36}(\mathbf{I}-3 \boldsymbol{n} \otimes \boldsymbol{n}), \quad \bar{l}(\xi)=\sqrt{f(\xi)} l, \quad f(\xi)=\frac{\xi^{2}(1+\exp (-\xi \pi))}{9+\xi^{2}} .
$$

Obviously the deviatoric part vanishes for a chaotic arrangement of the rods, $\xi=0$ and it turns into $\boldsymbol{J}_{0}^{\mathrm{dev}}=$ $l^{2} / 36(\mathbf{I}-3 \boldsymbol{n} \otimes \boldsymbol{n})$ if all the rods are aligned. It should be emphasized that by its very construction $\left\langle\boldsymbol{J}_{\infty}\right\rangle$ is the microinertia tensor on the continuum scale, however, after a very long time, so that there was ample of opportunity for the rods to arrange and find an equilibrium between the two concurring forces, the electric field, that wants them to align, and the disorientation effect due to a temperature different from absolute zero. We may refer to this by writing $\left\langle\boldsymbol{J}_{\infty}\right\rangle \equiv \boldsymbol{J}(t \rightarrow \infty)$.

We are now in a position to compare two homogenized lengths, first, $\left\langle l_{\infty}\right\rangle$ from Eq. (2.9) and, second, $\bar{l}(\xi)$ from Eq. $(2.11)_{3}$ as a function of the scattering parameter. The result is shown in Fig. 4. It is fair to say that the difference between the two approaches is small. The average length predicted by the microinertia approach is slightly less.

Moreover, we can compare the final result (2.11) with the microinertia tensor of a spheroidal ellipsoid with minor axes $a_{\infty}=b$ in $\boldsymbol{e}_{1}^{*}$ and $\boldsymbol{e}_{2}^{*}$ directions, respectively, and the major axes $c_{\infty}$ in $\boldsymbol{e}_{3}^{*} \equiv \boldsymbol{n}$ direction. It is given by:

$$
\boldsymbol{J}^{\mathrm{se}}=\boldsymbol{J}^{\mathrm{se}, \mathrm{sp}}+\boldsymbol{J}^{\mathrm{se}, \mathrm{dev}}
$$

with spherical and deviatoric parts,

$$
\boldsymbol{J}^{\mathrm{se}, \mathrm{sp}}=\frac{2}{15}\left(2 a_{\infty}^{2}+c_{\infty}^{2}\right) \mathbf{I}, \quad \boldsymbol{J}^{\mathrm{se}, \mathrm{dev}}=\frac{1}{15}\left(c_{\infty}^{2}-a_{\infty}^{2}\right)(\mathbf{I}-3 \boldsymbol{n} \otimes \boldsymbol{n}) .
$$

By comparison of both expressions we conclude that

$$
a_{\infty}^{2}=\frac{5}{36} l^{2}(1-f(\xi)), \quad c_{\infty}^{2}=\frac{5}{36} l^{2}(1+2 f(\xi))
$$

The $\infty$ signs indicate that we consider the situation after a long time. In the absence of an $\boldsymbol{E}$-field, i.e., for total chaos, we find $a_{\infty}=c_{\infty}$, and for complete orientation $a_{\infty}=0, c_{\infty}=\sqrt{5 / 12} l$, as it should be. The latter result had been obtained before in [21] in context with the concept of an equivalent rod length. On first glance it is surprising that $c_{\infty}=l / 2$ does not hold. This is because in an ellipsoid in contrast with the rod the mass must be considered as not evenly spread along the main axis.

Summarizing we may say that homogenization allowed us to analyze the final stage of the development of the microinertia. We now turn to the modeling of its temporal development. 


\subsection{The continuum model for the microinertia tensor}

Note that the spherical part of the microinertia tensor cannot change in time, because the microparticles in the representative volume are rigid. Hence, temporal development of anisotropy is only characterized by the deviatoric part of the microinertia tensor, $\boldsymbol{J}^{\mathrm{dev}}$. Consequently, the production term must be deviatoric, $\chi=\chi^{\mathrm{dev}}$, and be expressed in terms of deviators of microinertia. Hence, by using the nomenclature established in context with Eq. (2.11) we have

$$
\boldsymbol{J}=\boldsymbol{J}^{\mathrm{sp}}+\boldsymbol{J}^{\mathrm{dev}}, \quad \boldsymbol{J}^{\mathrm{sp}}=J_{0} \mathbf{I}, \quad J_{0}=\frac{l^{2}}{18}=\text { const. } \equiv \frac{2}{15}\left(2 a^{2}(t)+c^{2}(t)\right),
$$

and the balance (1.12) for the inertia tensor reduces to

$$
\frac{\mathrm{d} \boldsymbol{J}^{\mathrm{dev}}}{\mathrm{d} t}=\chi^{\mathrm{dev}}
$$

where

$$
\boldsymbol{J}^{\operatorname{dev}}=\frac{1}{15}\left(c^{2}(t)-a^{2}(t)\right)(\mathbf{I}-3 \boldsymbol{n} \otimes \boldsymbol{n}) .
$$

$a(t)$ is the semiaxis of the spheroidal ellipsoid in the plane of isotropy, $c(t)$ is the semiaxis of the spheroid in the direction of the external field, $\boldsymbol{n}$. They are time dependent as indicated.

The disappearance of the linear and angular velocity parts in Eq. (1.12) is worth a comment. First note that the translational velocity $v$ must be zero. To prove this we argue as follows: The body force is given by the resulting Coulomb force within the RVE of volume $V$, namely $\rho \boldsymbol{f}=N\left(q^{+}+q^{-}\right) \boldsymbol{E} / V$. However, it vanishes because $q^{-}=-q^{+}$. Moreover, the medium is quasi "dust." Hence the stress tensor $\sigma$ vanishes. Therefore, by virtue of Eq. (1.2) we must conclude that $\boldsymbol{v}=\mathbf{0}$. On the other hand and with this in mind, if we now look at Eq. (1.9), we must conclude that all the velocities $\boldsymbol{v}_{i}$ of the elementary particles are erratic and remain so during the polarization process. This is also understandable, because the Coulomb force on each of the micro-dipoles is also zero. Their centers will not be accelerated in the direction of the field $\boldsymbol{E}$ and, hence, no macroscopic velocity $\boldsymbol{v}$ will result.

Second, similar to the stress tensor, $\boldsymbol{\sigma}$, there will be no couple stress tensor $\boldsymbol{\mu}$ in Eq. (1.3). There are also no volume couples on the macrolevel, because they result from the vector product $\rho \boldsymbol{m}=\boldsymbol{P} \times \boldsymbol{E}$. However, both $\boldsymbol{P}$ and $\boldsymbol{E}$ align with $\boldsymbol{n}$, so that the vector product vanishes. To put it differently: We would expect the dipole macroparticle to rotate only if is misaligned with respect to the electric field, so that a moment couple is created. But this is not the case in our arrangement.

Nevertheless, this still leaves the term $-\boldsymbol{\omega} \times \boldsymbol{J} \cdot \boldsymbol{\omega}$ in Eq. (1.3), and in it obviously only the deviatoric part $J^{\text {dev }}$ might contribute to a temporal development of the angular velocity $\omega$ :

$$
\boldsymbol{J} \cdot \frac{\mathrm{d} \boldsymbol{\omega}}{\mathrm{d} t}=-\boldsymbol{\omega} \times \boldsymbol{J}^{\mathrm{dev}} \cdot \boldsymbol{\omega} .
$$

This equation is identically satisfied and does not lead to internal contradictions, if for the physical reasons presented above $\boldsymbol{\omega}=\mathbf{0}$ is set. Unlike the case of the microvelocities $\boldsymbol{v}_{i}$ we cannot support this conjecture from Eq. (1.8) where the angular momenta of the microparticles were averaged. Indeed, we can represent the angular velocity $\boldsymbol{\omega}_{i}$ of a microparticle in the eigensystem of its inertia tensor, $\boldsymbol{\omega}_{i}=\omega_{i, j} \boldsymbol{e}_{i, j}^{*}$. Then according to Eqns. (2.4), (1.8) and (1.10) we find:

$$
\boldsymbol{\omega}=\left(N \mathbf{I}-\sum_{i=1}^{N} \boldsymbol{e}_{i, 3}^{*} \otimes \boldsymbol{e}_{i, 3}^{*}\right)^{-1} \cdot \sum_{i=1}^{N}\left(\boldsymbol{\omega}_{i}-\omega_{i, 3} \boldsymbol{e}_{i, 3}^{*}\right) .
$$

However, each microdipole will be subjected to a non-vanishing moment because of the applied electric field so that its angular velocity $\boldsymbol{\omega}_{i}$ will develop in time in contrast with its translational velocity $\boldsymbol{v}_{i}$.

The production $\chi^{\mathrm{dev}}$ is additively split into two contributions, one to account for the effect of the external field, called $\chi_{E}$, and one to account for the impact of temperature, called $\chi_{T}$, as follows:

Thermal polarization occurs rather slowly. In a constant external field equilibrium is established after some time $\tau_{p}$, which is known as the relaxation time of the polarization process. That is, a steady state during thermal polarization occurs when the external influence is compensated by internal thermal movement. Thus, 


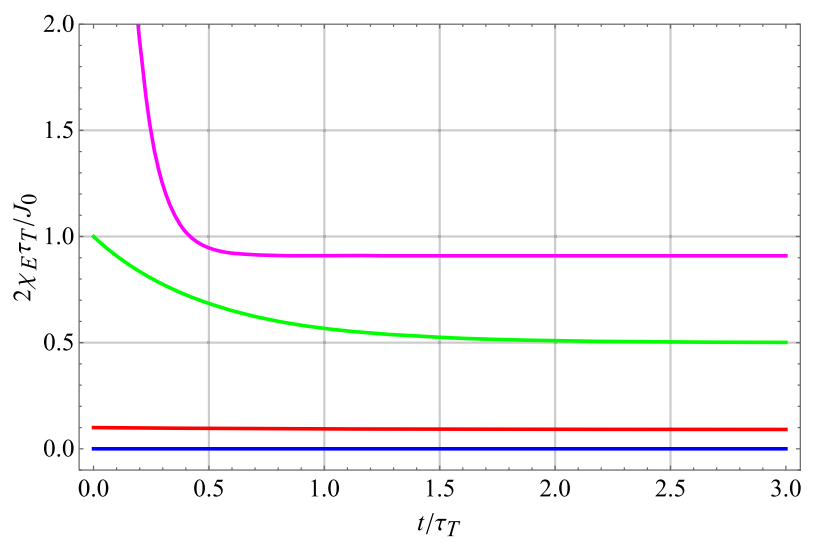

Fig. 5 Change of the source term $\chi_{E}$ vs. ${ }^{t} / \tau_{T}$ for $\tau_{T} / \tau_{E}=0.0001$ (blue), 0.1 (red), 1 (green), 10 (magenta)

the production term consists of a part that corresponds to the alignment of all microparticles in the direction of the external field without thermal movement,

$$
\chi_{E}(t)=\frac{1}{\tau_{E}(E)}\left(\boldsymbol{J}_{\infty}^{\mathrm{dev}}-\boldsymbol{J}^{\mathrm{dev}}(t)\right),
$$

where $\boldsymbol{J}_{\infty}^{\mathrm{dev}}=l^{2} / 36(\mathbf{I}-3 \boldsymbol{n} \otimes \boldsymbol{n})$ is the deviator of the microinertia that would be obtained if the electric field is infinitely strong and all microparticles would be forcefully aligned, $c p$., Eq. $(2.11)_{2}$ for $\xi \rightarrow \infty$. $\boldsymbol{J}^{\mathrm{dev}}(t)$ will strive toward this value but never quite reach it. $\tau_{E}$ is a (positive) relaxation time decreasing with increasing $E_{0}$ and characteristic of the intensity of the external effect, in agreement with experimental evidence. All of this indicates that the production $\chi_{E}$ has the character of a source, i.e., it is always positive.

The second part of the production characterizes the thermal movement,

$$
\chi_{T}(t)=-\frac{1}{\tau_{T}(T)} J^{\operatorname{dev}}(t),
$$

where $\tau_{T}$ is the time it takes for the material to return to an isotropic state due to temperature when the external field is turned off. The smaller the $\tau_{T}$-value, the faster the complete disorientation of the microparticles. Since the disorientation of particles is associated with thermal motion, $\tau_{T}$ should be a decreasing function with temperature. Note that the production $\chi_{T}$ is actually a sink term, since it is always less or at most equal to $\mathbf{0}$, i.e., essentially negative.

The integration of Eq. (2.16) with the initial condition $\boldsymbol{J}^{\mathrm{dev}}=\mathbf{0}$ yields the temporal development of the inertia tensor:

$$
\boldsymbol{J}(t)=J_{0} \mathbf{I}+J_{\infty}\left[1-\exp \left(-\frac{t}{\tau_{p}}\right)\right](\mathbf{I}-3 \boldsymbol{n} \otimes \boldsymbol{n}), \quad J_{\infty}=\frac{l^{2}}{36} \frac{\tau_{T}}{\tau_{E}+\tau_{T}}, \quad \tau_{p}=\frac{\tau_{E} \tau_{T}}{\tau_{E}+\tau_{T}} .
$$

In here $J_{\infty}$ denotes the limit value of the moment of inertia for $t \rightarrow \infty$. Note that the stationary value of (2.22) for $t \rightarrow \infty$ does not coincide with the moment of inertia of the microparticle (2.5). The difference is the factor $\frac{\tau_{T}}{\tau_{E}+\tau_{T}}$. This makes sense because not all of the dipoles are aligned in the $\boldsymbol{n}$-direction due to thermal motion. Thus, this quantity characterizes the equilibrium distribution of dipoles over orientations. In fact by comparison with (2.11) we must conclude that:

$$
\frac{\tau_{T}}{\tau_{E}+\tau_{T}} \equiv f(\xi)=\frac{\xi^{2}(1+\exp (-\xi \pi))}{9+\xi^{2}} .
$$

Consider the first limit case, which is $\xi=0$, i.e., total disorder of the particles. Then the right hand side of (2.23) is equal to zero. Consequently, $\tau_{T} \rightarrow 0$ and the thermal sink term, $\chi_{T}$, creating chaos will dominate. The second case, total alignment, results for $\xi \rightarrow \infty$. Then the right-hand side of (2.23) is equal to one, which is achieved for $\tau_{E} \rightarrow 0$. Then the influence of the $\boldsymbol{E}$-field related source term, $\chi_{E}$, is dominant.

Figure 5 illustrates the behavior of the factor in front of the normalized source term (2.20), i.e., essentially the component $\boldsymbol{e}_{1} \cdot \chi_{E} \cdot \boldsymbol{e}_{1}\left(\boldsymbol{e}_{1} \perp \boldsymbol{n}\right)$ vs. normalized time $t / \tau_{T}$. Recall that if $\tau_{T} / \tau_{E}<<1$, then the source term 


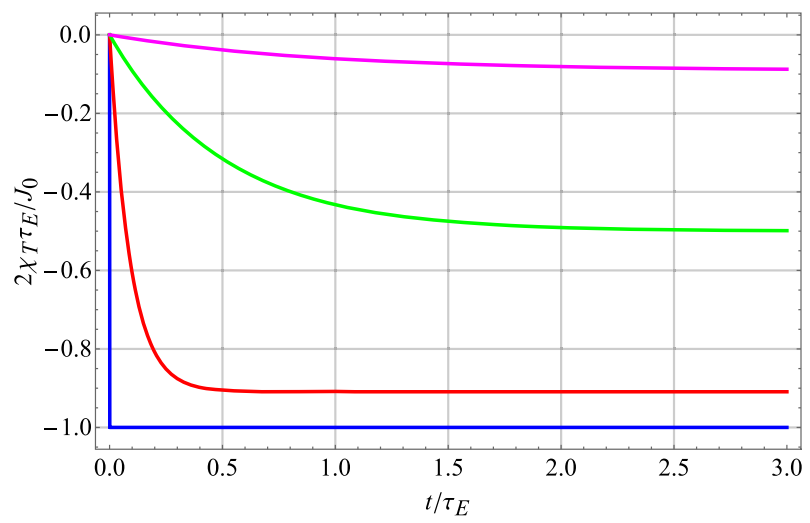

Fig. 6 Change of the sink term $\chi_{T}$ vs. $t / \tau_{T}$ for $\tau_{T} / \tau_{E}=0.0001$ (blue), 0.1 (red), 1 (green), 10 (magenta)

should be small, because the chaotic effect of temperature will overcome the ordering imposed by the electric field. This is demonstrated by the blue and red lines. More mathematically speaking, we find that if $\tau_{T} / \tau_{E} \rightarrow 0$ then $2 \chi_{E} \tau_{T} / J_{0} \rightarrow 0$, i.e., temperature dominance, and if $\tau_{T} / \tau_{E} \rightarrow \infty$, then $2 \chi_{E} \tau_{T} / J_{0} \rightarrow 1$, i.e., electric field dominance.

Figure 6 illustrates the behavior of the analogous factor in front of the normalized sink term (2.21) vs. normalized time $t / \tau_{E}$. Again recall that if $\tau_{T} / \tau_{E}<<1$, then the absolute value of the sink term should be large, because the chaotic effect of temperature will overcome the ordering imposed by the electric field. This is demonstrated by the blue and red lines. Moreover, we find that if $\tau_{T} / \tau_{E} \rightarrow 0$ then $2 \chi_{T} \tau_{E} / J_{0} \rightarrow-1$, i.e., temperature dominance, and if $\tau_{T} / \tau_{E} \rightarrow \infty$, then $2 \chi_{E} \tau_{E} / J_{0} \rightarrow 1$, i.e., electric field dominance.

It should also be noted that by comparison of the result shown in (2.22) with (2.15) 3 and (2.17) we find for the semi-axes:

$$
\begin{aligned}
& a(t)^{2}=\frac{5}{36} l^{2}\left[1-\frac{\tau_{T}}{\tau_{E}+\tau_{T}}\left(1-\exp \left(-\frac{\tau_{E}+\tau_{T}}{\tau_{E} \tau_{T}} t\right)\right)\right], \\
& c(t)^{2}=\frac{5}{36} l^{2}\left[1+2 \frac{\tau_{T}}{\tau_{E}+\tau_{T}}\left(1-\exp \left(-\frac{\tau_{E}+\tau_{T}}{\tau_{E} \tau_{T}} t\right)\right)\right] .
\end{aligned}
$$

We conclude that:

$$
a_{\infty}^{2}=\frac{5}{36} l^{2} \frac{\tau_{E}}{\tau_{E}+\tau_{T}}, \quad c_{\infty}^{2}=\frac{5}{36} l^{2} \frac{\tau_{E}+3 \tau_{T}}{\tau_{E}+\tau_{T}} .
$$

This result is consistent with Eqs. (2.14) and (2.23). Also in the case of temperature supremacy $\tau_{T} / \tau_{E} \rightarrow 0$ it follows that $a_{\infty}=c_{\infty}$, in other words isotropy.

The deviator of the time-developing microinertia (2.22) can also be written in the form:

$$
\boldsymbol{J}(t)^{\operatorname{dev}}=\frac{\bar{l}_{\tau}(t)^{2}}{36}(\mathbf{I}-3 \boldsymbol{n} \otimes \boldsymbol{n}),
$$

where an effective length $\bar{l}_{\tau}(t)$ was defined:

$$
\bar{l}_{\tau}(t)=\sqrt{\frac{\tau_{T}}{\tau_{E}+\tau_{T}}\left[1-\exp \left(-\frac{\tau_{E}+\tau_{T}}{\tau_{E} \tau_{T}} t\right)\right]} l .
$$

Obviously we obtain for very long times:

$$
\bar{l}_{\tau}(\infty)=\sqrt{\frac{\tau_{T}}{\tau_{E}+\tau_{T}}} l,
$$

and this result is consistent with $(2.11)_{3,4}$ and (2.23). The stationary length is plotted in Fig. 7. Clearly, for dominant thermal disorder $\left(\tau_{T}<<\tau_{E}\right)$ the effective length goes to zero and chaos prevails, whereas with a strong electric field $\left(\tau_{E}<<\tau_{T}\right)$ saturation can be reached. It is curious to note that the average length is not equal to 0.5 for $\tau_{E}=\tau_{T}$ but rather to the square root of it. Mathematically speaking this is due to the equation of length identification (2.27). The microinertia itself favors the square. 


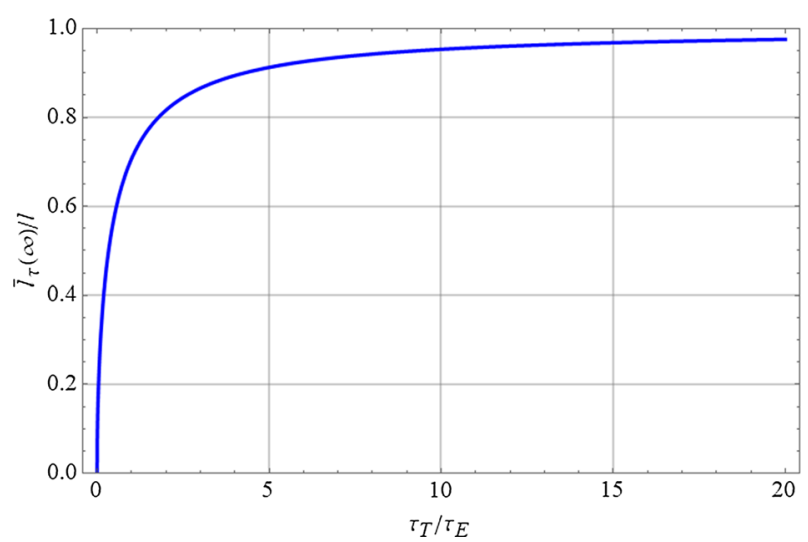

Fig. 7 Stationary average length $l_{\tau}(\infty)$ as a function of $\tau_{T} / \tau_{E}$

\subsection{Alternating electric field and dispersion}

It was emphasized in context with Eq. (2.20) that the relaxation time $\tau_{E}$ depends on the electric field $\boldsymbol{E}$. More specifically it decreases with increasing electric field. So far $\tau_{E}$ was treated as a constant. If we now wish to study the impact of an electric field alternating in magnitude but not changing its direction in space on the development of the microinertia tensor and therefore on the development of polarization, consequently, we have to allow that $\tau_{E}$ changes with time. Let us write:

$$
\tau_{E}(t)=\frac{\tau_{E}^{0}}{\cos ^{2}(\omega t)},
$$

where $\tau_{E}^{0}$ is a true constant. Because of the square we do not repolarize, however, the ordering effect of the electric field will not be optimal at all times. The cosine function was chosen for pure convenience. It is a smooth function and leads to no problems during the numerical integration. Then the thermal production shown in Eq. (2.21) will take over even more and has additional opportunity to reestablish chaos. Consequently, the effective length $\bar{l}_{\tau}$ and thus the molecular polarization will fluctuate more or less heavily depending on the chosen frequency $\omega$. Such a dependence on frequency is known as dispersion. Moreover, between the applied alternating electric field and the varying polarization there may also be a phase shift.

If Eq. (2.29) is used in context with (2.20) and (2.21), the differential equation (2.16) can numerically be solved: Fig. 8 shows the effective length as a function of normalized time for $\tau_{T} / \tau_{E}^{0}=0.1$ (top left), 1.0 (top right), 5.0 (bottom left), 10.0 (bottom right) for four choices of normalized frequency $\omega / \tau_{T}=0$ (blue), 1 (red), 2 (green), and 5 (magenta). Of course, just numerical solutions of the differential equation (2.16) are possible in the alternating case. The following is observed:

- The smaller $\tau_{T} / \tau_{E}^{0}$, the smaller the effective polarization $l_{\tau}$, because then the thermal effect prevails (resulting in smaller values of $\tau_{T}$ ) or the electric field is too weak (resulting in larger values of $\tau_{E}$ ).

- The undulating polarization curves stay below the blue one for the non-alternating electric field. In other words, the thermal effect is more effective.

- The larger the frequency of the $\omega$ of the alternating electric field, the closer the effective polarization comes to the one when the electric field does not alternate: The temperature has less time to take effect and dispersion is strongly visible. However, even at very high frequencies the effective polarization does not quite reach the one for a non-alternating field.

The effect of dispersion is further analyzed in Fig. 9. The curve and corresponding dots are for $\tau_{T} / \tau_{E}^{0}=0.2$ (red), 0.5 (green), 1.0 (blue), 2.0 (black), and 10.0 (magenta). Note that:

- Increasing $\omega \tau_{T}$ from zero results in an initially steep decline followed by an increase that finally leads to saturation.

- The higher the $\tau_{T} / \tau_{E}^{0}$, the smaller the difference between the value for polarization at $\omega \tau_{T}=0$ and the saturation level. The particles have time to adjust. However, the average length of the non-transient case is never fully reached.

- The dispersion effect becomes more pronounced if $\tau_{T} / \tau_{E}^{0}$ is increased. 

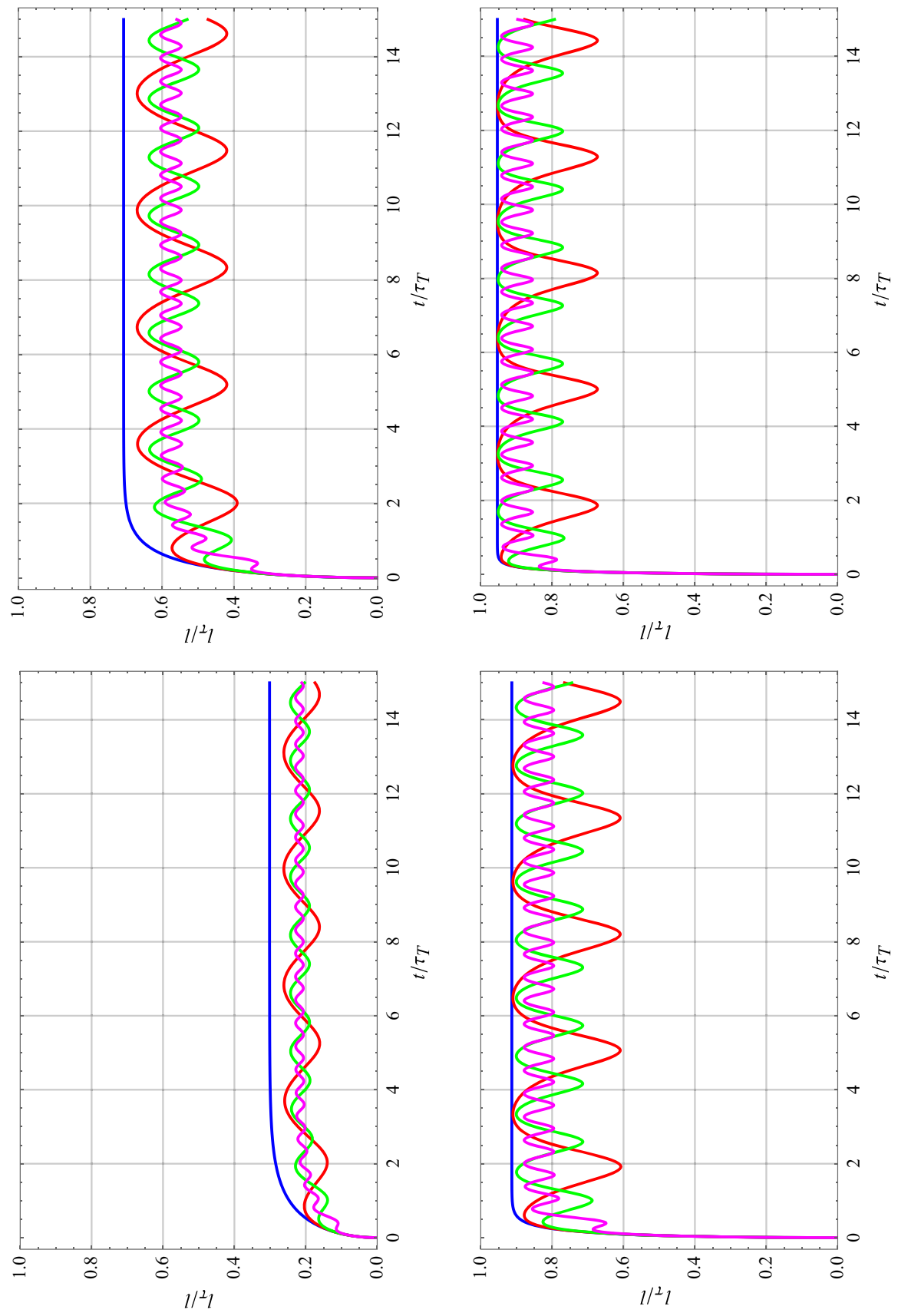

苍 


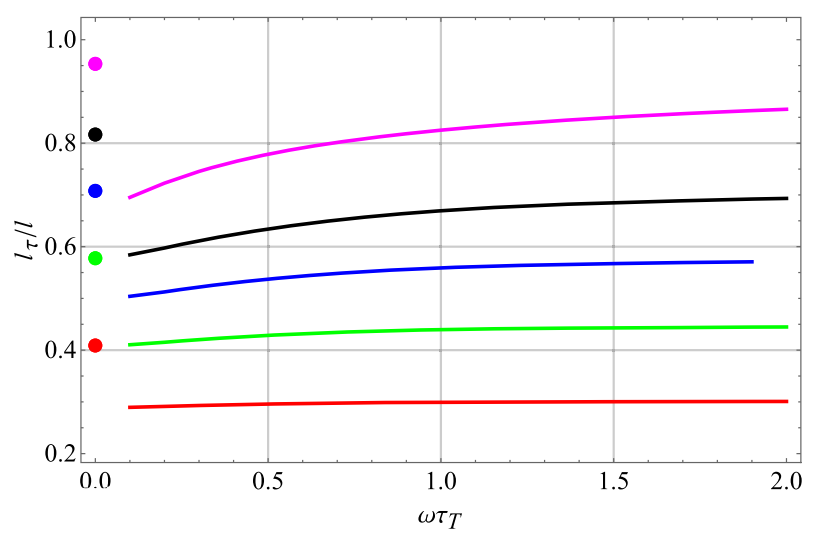

Fig. 9 Effective polarization $l_{\tau}$ as a function of normalized frequency of the electric field (see text)

\section{Conclusions and outlook}

In this paper the following were achieved:

- The importance of micropolar media for studying electric phenomena, such as polarization was emphasized, because they allow modeling materials with an internal structure.

- The phenomenon of dielectric polarization was discussed in terms of micromodels. In particular the microphenomenon of orientational polarization was linked to the continuum level from a new viewpoint, namely to an extended version of micropolar theory.

- The process of developing orientational polarization was analyzed by introducing two production terms in the extended balance of microinertia, one for the chaotic effect of temperature and one for the ordering effect of the electric field.

- The final stage of polarization obtained from the microinertia model was compared to results obtained from homogenization both traditionally by calculating the effective length of oriented rigid dipoles and non-traditionally by calculating their average inertia tensor.

It is fair to say that the homogenization technique applied to orientational polarization in this paper captures the essential physics features of this process in a novel way, but it seems rather crude when compared to more sophisticated methods, for example the ones described in [18]. The authors of that paper focus on the electromagnetic side of the problems; in other words, they consider polarization and magnetization in terms of Maxwell's equations. The applications of their techniques to the mechanical viewpoint of extended micropolar media should be subject of further studies.

Further research in the field of extended micropolar theory will surely include a similar investigation of the magnetic susceptibility. Both will potentially lead to an investigation of cross-property connections as follows. Note that the classical approaches, which are based on averaging according to $\langle p\rangle=q\langle l\rangle$, allow only the polarization of the material to be estimated. In contrast with that the approach proposed in this work, associated with a change in the tensor of inertia at the macrolevel, makes it possible to establish relationships between various physical and mechanical processes. In particular the reorientation of microparticles from a chaotic to an ordered state transforms an initially isotropic heterogeneous material into a transversally isotropic one, which, in turn, leads to a change in the effective elastic, or conductive (electro, heat, diffusion) properties of the material.

In the future our research might also be helpful for modeling structural transitions in nematic crystals. Surely, the interaction between the microparticles in such materials will be more complicated than the dust type of interaction that was assumed in our present work. Nevertheless, the orientational transitions under the action of electro-magnetic fields are similar to ours, as can be seen in a recent publication, where such modeling was attempted numerically [29]. If such coupled problems of nematic crystals are studied, it will also become necessary to reconsider the balance of internal energy (1.4) to account for effects of electro-magnetic dissipation (Joule heating).

Also a remark should be made regarding the attempts of researchers to include elastic effects to dielectrics. As it was mentioned so far we did not study truly coupled interaction between all field equations. Instead we specialized to "dust," which led to a balance of spin that was identically satisfied. The foundations of a more 
sophisticated electro-micropolar theory were laid very early in a seminal paper by Dixon and Eringen [6]. The methods explained in there now need to be used in the extended version of micropolar media. In particular it will be worthwhile to address the question as to whether the idea of higher electric moments (quadropoles) are in some way equivalent or go beyond to our idea of a dynamic microinertia following its own balance.

In the same context it should also be asked as to whether the concept of micromorphic continua is equivalent or goes beyond our concept of a microinertia modeling structural change. A starting point could be a comparison with results from [25-27], and [28]. To begin with it will become necessary to discuss the differences between the microinertia tensor of micromorphic continua and our microinertia for rigid body points. Then the striking mathematical similarity between the former and the quadropole tensor must be examined and, finally, the question must be answered as to whether the production of our microinertia describing structural change can be linked to the additional freedom of deformation inherent to micromorphic continua.

Funding Open Access funding enabled and organized by Projekt DEAL.

Open Access This article is licensed under a Creative Commons Attribution 4.0 International License, which permits use, sharing, adaptation, distribution and reproduction in any medium or format, as long as you give appropriate credit to the original author(s) and the source, provide a link to the Creative Commons licence, and indicate if changes were made. The images or other third party material in this article are included in the article's Creative Commons licence, unless indicated otherwise in a credit line to the material. If material is not included in the article's Creative Commons licence and your intended use is not permitted by statutory regulation or exceeds the permitted use, you will need to obtain permission directly from the copyright holder. To view a copy of this licence, visit http://creativecommons.org/licenses/by/4.0/.

\section{References}

1. Abali, B.E., Reich, F.A.: Thermodynamically consistent derivation and computation of electro-thermo-mechanical systems for solid bodies. Comput. Methods Appl. Mech. Eng. 319, 567-595 (2017)

2. Alessandroni, S., Andreaus, U., Dell'Isola, F., Porfiri, M.: Piezo-electromechanical (pem) Kirchhoff-love plates. Eur. J. Mech. A/Solids 23(4), 689-702 (2004)

3. Altenbach, H., Eremeyev, V.A., Lebedev, L.P., Rendón, L.A.: Acceleration waves and ellipticity in thermoelastic micropolar media. Arch. Appl. Mech. 80(3), 217-227 (2010)

4. Altenbach, J., Altenbach, H., Eremeyev, V.A.: On generalized cosserat-type theories of plates and shells: a short review and bibliography. Arch. Appl. Mech. 80(1), 73-92 (2010)

5. Chen, K.: Microcontinuum balance equations revisited: the mesoscopic approach. J. Non-Equilib. Thermodyn. 32, 435-458 (2007)

6. Dixon, R.C., Eringen, A.C.: A dynamical theory of polar elastic dielectrics-I. Int. J. Eng. Sci. 3(3), 359-377 (1965)

7. Dłużewski, P.H.: Finite deformations of polar elastic media. Int. J. Solids Struct. 30(16), 2277-2285 (1993)

8. Eringen, A.C.: Continuum Physics, vol. IV. Academic Press, New York (1976)

9. Eringen, A.C.: A unified continuum theory of electrodynamics of liquid crystals. Int. J. Eng. Sci. 35(12/13), 1137-1157 (1997)

10. Eringen, A.C.: Microcontinuum Field Theory I, Foundations and Solids. Springer, New York (1999)

11. Eringen, A.C., Kafadar, C.B.: Polar field theories. Continuum physics IV. Academic Press, London (1976)

12. Giorgio, I., Culla, A., Del Vescovo, D.: Multimode vibration control using several piezoelectric transducers shunted with a multiterminal network. Arch. Appl. Mech. 79(9), 859-879 (2009)

13. Ivanova, E., Vilchevskaya, E., Müller, W.H.: Time derivatives in material and spatial description-What are the differences and why do they concern us? In: Naumenko, K., Aßmus, M. (eds.) Advanced Methods of Mechanics for Materials and Structures, pp. 3-28. Springer, New York (2016)

14. Ivanova, E.A., Vilchevskaya, E.N.: Micropolar continuum in spatial description. Continuum Mech. Thermodyn. 28(6), 1759-1780 (2016)

15. Kachanov, M., Sevostianov, I.: Micromechanics of Materials, with Applications. Springer, Cham (2018)

16. Kestelman, V.N., Pinchuk, L.S., Goldade, V.A.: Electrets in engineering: fundamentals and applications. Springer, Cham (2013)

17. Lee, K.W., Lee, C.E.: Strain-induced topological phase transition with inversion of the in-plane electric polarization in tiny-gap semiconductor sige monolayer. Sci. Rep. 10(1), 1-10 (2020)

18. Mazur, P., Nijboer, B.R.A.: On the statistical mechanics of matter in an electromagnetic field I: Derivation of the maxwell equations from electron theory. Physica 19(1-12), 971-986 (1953)

19. Mindlin, R.D.: Micro-structure in linear elasticity. Arch. Rat. Mech. Anal. 16(1), 51-78 (1964)

20. Morozova, A.S., Vilchevskaya, E.N., Müller, W.H., Bessonov, N.M.: Interrelation of heat propagation and angular velocity in micropolar media. In: Altenbach, H., Belyaev, A., Eremeyev, V.A., Krivtsov, A., Porubov, A.V. (eds.) Dynamical Processes in Generalized Continua and Structures, pp. 413-425. Springer, Cham (2019)

21. Müller, W.H., Vilchevskaya, E.N.: Micropolar theory with production of rotational inertia: a rational mechanics approach. In: Altenbach, H., Pouget, J., Rousseau, M., Collet, B., Michelitsch, T. (eds.) Generalized Models and Non-classical Approaches in Complex Materials 1, pp. 195-229. Springer, Cham (2018) 
22. Müller, W.H., Vilchevskaya, E.N., Weiss, W.: A meso-mechanics approach to micropolar theory: a farewell to material description. Phys. Mesomech. 20(3), 13-24 (2017)

23. Oevel, W., Schröter, J.: Balance equation for micromorphic materials. J. Stat. Phys. 25(4), 645-662 (1981)

24. Resta, R., Vanderbilt, D.: Theory of polarization: a modern approach. In: Ahn, C., Rabe, K., Triscone, J. (eds.) Physics of Ferroelectrics, pp. 31-68. Springer, Berlin, Heidelberg (2007)

25. Romeo, M.: Micromorphic continuum model for electromagnetoelastic solids. Z. Angew. Math. Phys. 62(3), 513-527 (2011)

26. Romeo, M.: Micromorphic elastic dielectrics: linear model and micropolar isotropic thin layers. Int. J. Solids Struct. 49(26), 3935-3941 (2012)

27. Romeo, M.: Polarization in dielectrics modeled as micromorphic continua. Z. Angew. Math. Phys. 66(3), 1233-1247 (2015)

28. Romeo, M.: A microstretch continuum approach to model dielectric elastomers. Z. Angew. Math. Phys. 71(2), 1-13 (2020)

29. Rosi, G., Placidi, L., Dell'Isola, F.: "Fast" and "slow" pressure waves electrically induced by nonlinear coupling in Biot-type porous medium saturated by a nematic liquid crystal. ZAMP 68(2), 51-64 (2017)

30. Sawada, A.: Dielectric process of space-charge polarization for an electrolytic cell with blocking electrodes. J. Chem. Phys. 129(6), 064,701-064,7012 (2008)

31. Sutter, P.H., Nowick, A.S.: Ionic conductivity and time-dependent polarization in NaCl crystals. J. Appl. Phys. 34(4), 734-746 (1963)

32. Truesdell, C., Toupin, R.A.: The classical field theories. Springer, Heidelberg (1960)

33. Vilchevskaya, E.: Micropolar theory with inertia production. In: Altenbach, H., Öchsner, A. (eds.) Advanced Structured Materials, vol. 100, pp. 421-442. Springer Nature, Cham (2019)

34. Yildizdag, M.E., Tran, C.A., Barchiesi, E., Spagnuolo, M., dell'Isola, F., Hild, F.: A multi-disciplinary approach for mechanical metamaterial synthesis: a hierarchical modular multiscale cellular structure paradigm. In: Altenbach, H., Öchsner, A. (eds.) State of the Art and Future Trends in Material Modeling, pp. 485-505. Springer, Cham (2019)

35. You, K., Goudos, S.K.: Materials characterization using microwave waveguide system. In: Goudos, S.K. (ed.) Microwave Systems and Applications, pp. 341-358. IntechOpen, London (2017)

Publisher's Note Springer Nature remains neutral with regard to jurisdictional claims in published maps and institutional affiliations. 